\title{
Bilateral internal carotid artery dissection associated with prior syphilis: a case report and review of the literature
}

This article was published in the following Dove Press journal:

Neuropsychiatric Disease and Treatment

10 June 2016

Number of times this article has been viewed

\author{
Antonio Marangi' \\ Giuseppe Moretto 2 \\ Manuel Cappellari ${ }^{2}$ \\ Nicola Micheletti ${ }^{2}$ \\ Giampaolo Tomelleri² \\ Paolo Bovi ${ }^{2}$ \\ 'Section of Neurology, Department \\ of Neurosciences, Biomedicine and \\ Movement Sciences, Policlinico G.B \\ Rossi, University of Verona, Verona, \\ ${ }^{2}$ Stroke Unit, Division of Neurology, \\ Azienda Ospedaliera Universitaria \\ Integrata Verona, Verona, Italy
}

\begin{abstract}
Bilateral internal carotid artery dissection is a rare entity, and its presentation may include cerebral ischemia. We describe the case of a 69 -year-old man with ischemic stroke and radiological evidence of intimal flap of both internal carotid arteries suggestive for dissection. During the hospitalization, our patient was found positive for a previous syphilis infection. We conducted a review of the literature, with evidence of a few cases of ischemic stroke presumably related to a prior syphilis. The absence of major cardiovascular risk factors in our patient leads us to believe that an etiopathogenetic link may exist between these two conditions.
\end{abstract}

Keywords: bilateral carotid dissection, ischemic stroke, neurosyphilis

\section{Introduction}

Bilateral internal carotid artery dissection (ICAD) is a rare entity, and its clinical presentation may include cerebral ischemia. ${ }^{1}$ On the other hand, syphilis represents a global disease, with $\sim 10.6$ million new cases reported in 2005 and 2008 throughout the world. ${ }^{2}$ It remains prevalent in many developing countries (such as southern Asia and sub-Saharan Africa), but it has reemerged as a health concern in many developed nations in the past 10 years. We herein report the case of a 69-year-old man with ischemic stroke and radiological evidence of bilateral ICAD presumably due to a prior syphilis infection.

\section{Case report}

The current study respected the ethical principles depicted in the Declaration of Helsinki, and written informed consent was obtained from the patient permitting their case be published in this report. A 69-year-old man, previously fit and well, presented to the local emergency department $\sim 120$ minutes after developing weakness in the left limbs and slurred speech. Clinical examination revealed plegia of the left arm with paresis of the homolateral leg and mild left central facial palsy; there was also a left tactile hemihypesthesia with extinction at the double simultaneous tactile stimulation. A left homonymous hemianopsia was also observed. There was no complaint of headache or cervical pain. He was found to have normal vital signs, and laboratory studies revealed normal peripheral cell counts, electrolytes, and coagulation values. A noncontrast head computed tomography scan was unremarkable. The patient was then transferred to our stroke unit with a maximum National Institutes of Health Stroke Scale (NIHSS) score of 9. After obtaining written informed consent, intravenous recombinant tissue plasminogen activator was administered per standard
Correspondence: Antonio Marangi Department of Neurosciences, Biomedicine and Movement Sciences, University of Verona, Policlinico Borgo Roma, Piazzale LA Scuro I0, Verona 37| 34 , Italy

Tel +39334355 5087

Fax +39045802 7492

Email marangi2010@libero.it 


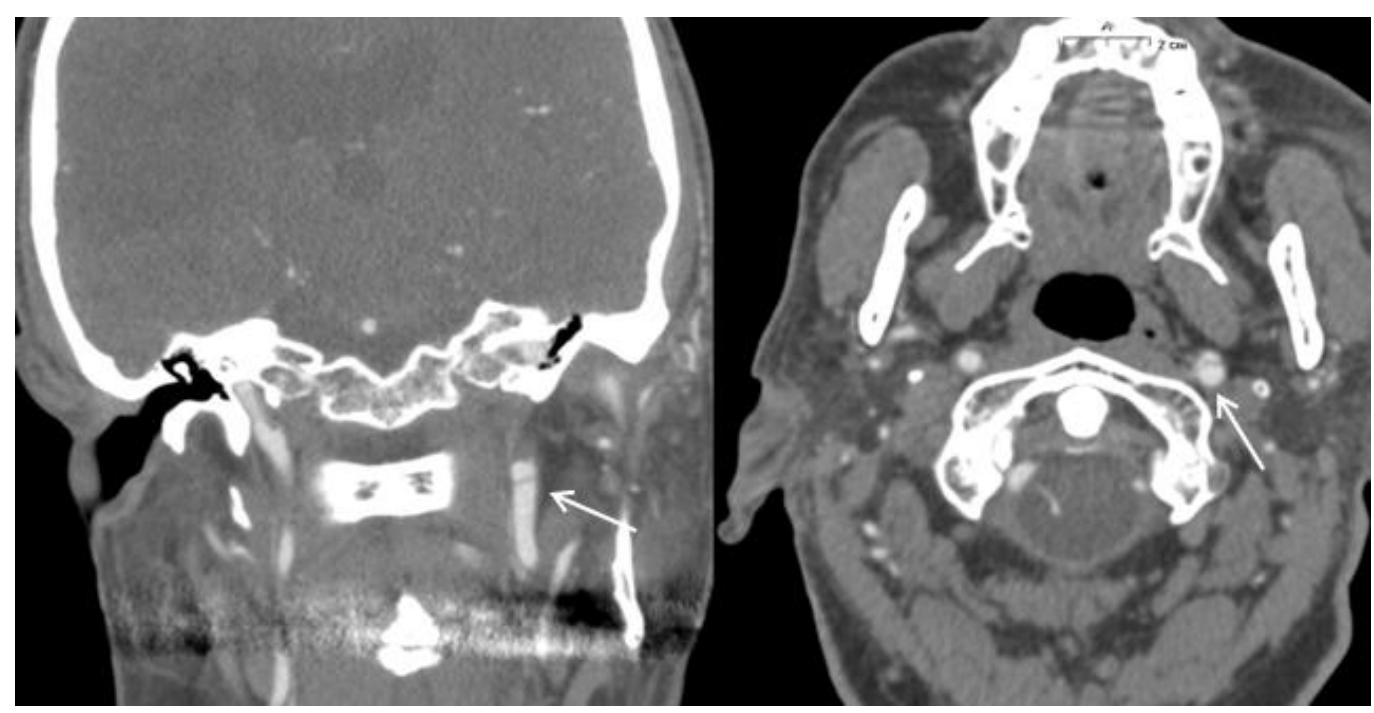

Figure I Coronal (left) and axial (right) computed tomography angiographic images showing an intimal flap of the left internal carotid artery (white arrows).

protocol $(0.9 \mathrm{mg} / \mathrm{kg}$ over 60 minutes as a $10 \%$ bolus and $90 \%$ infusion). The following morning, the neurological examination showed a slight worsening (NIHSS score of 11), with evidence of a left hemispatial neglect. A noncontrast computed tomography scan revealed an area of hypodensity in the right parietooccipital region; no hemorrhage was seen. The patient was then placed on antiplatelet therapy (aspirin $100 \mathrm{mg}$ once daily) with high-dose statin (atorvastatin $80 \mathrm{mg}$ once daily). Our patient did not have a history of diabetes and/or arterial hypertension. Blood tests, including a complete lipid profile, were normal, except for a mild hyperhomocysteinemia $(19.9 \mu \mathrm{mol} / \mathrm{L}$ with an upper normal limit $<15$ ). A transthoracic echocardiogram turned out to be unremarkable. Continuous monitoring of cardiac activity did not detect episodes compatible with atrial fibrillation. Carotid doppler revealed a small plaque at the right carotid bulb. Hence, a computed tomography angiography of intra- and extracranial vessels was performed, which revealed a bilateral intimal flap in the intracranial portion of internal carotid artery that was also confirmed by a further computed tomography angiography control, with no evidence of intramural hematomas; this finding was compatible with bilateral dissection (Figures 1 and 2). A T1-weighted with fat saturation magnetic resonance imaging was not diagnostic, owing to motion artifacts. During the hospitalization, the patient reported some business trips to South America in the past 10 years. Just before discharge, treponemal serologic tests were performed with a positive Treponema pallidum particle

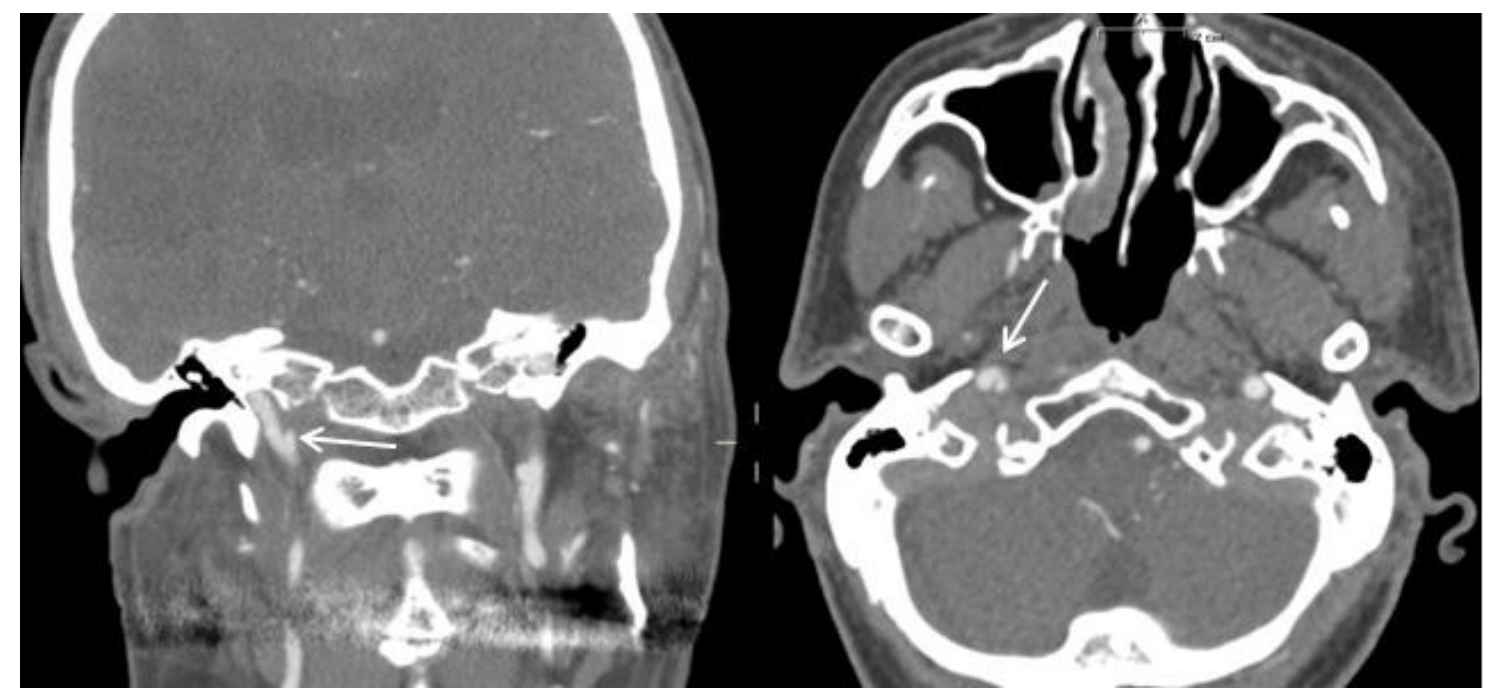

Figure 2 Coronal (left) and axial (right) computed tomography angiography scans showing a wispy intimal flap in the right internal carotid artery (white arrows). 
agglutination assay (TP-PA) result of 1:1,280, while venereal disease research laboratory test (VDRL) turned out to be negative. According to our consultant in infectious diseases, this serological profile had to be considered compatible with a previously treated infection with T. pallidum (confirmed later by the same patient, who did not follow-up any other serologic test after the antibiotic treatment of the infection nor any other examination with an infectious diseases specialist); therefore, there was no indication for further investigations (eg, lumbar puncture for cerebrospinal fluid VDRL) and/ or for an antibiotic treatment. HIV test was not performed because the patient did not give his informed consent.

In the next few days, the patient's neurological examination was stable. He was then transferred to an outlying rehabilitation facility.

\section{Discussion}

Bilateral ICAD is a rare entity, representing 5\%-28\% of all dissections. ${ }^{1}$ The cause is often not found. Many conditions, including some genetic diseases, have been postulated to play a role in unilateral ICAD, and this has been widely described in the literature. Our patient did not have a family history of ischemic stroke. He did not present highly stretchy skin, joint hypermobility, or marfanoid habitus. Fibromuscular dysplasia has been associated with up to $50 \%$ of bilateral dissections, but this was not detected by the imaging in our case. ${ }^{3}$ A mild hyperhomocysteinemia, as detected in our patient, could be considered as a risk factor by means of damage to arterial wall. ${ }^{4}$ Among the other typical risk factors for cervical artery dissection, there was no history of head or cervical trauma in the recent past..$^{5}$ Moreover, there was no previous or active drug abuse.

In our patient, we found a serologic positivity for a prior syphilis infection. Ischemic stroke may be particularly seen in meningovascular syphilis, which is defined as acute focal neurological impairment in the territory of a vessel in the brain or spinal cord due to syphilis as determined by clinical examination and/or neurological imaging, in the absence of cardiac embolism and/or supra-aortic vessel stenosis. ${ }^{6}$ This phase manifests 5-12 years after initial infection with T. pallidum. ${ }^{7}$ Vascular events in meningovascular syphilis are related to endarteritis of vessels anywhere in the central nervous system. Lymphocytic infiltrates and fibrous intimal proliferation lead to occlusion of several types of arteries, including medium caliber intracranial arteries, meningeal branches, and large arteries at the base of the brain. ${ }^{8}$ In this scenario, carotid dissection represents a rare but possible consequence of such arteriopathic process. ${ }^{9}$
In our case, the patient's history, combined with the absence of clear additional cardiovascular risk factors, leads us to believe that bilateral ICAD can be related to the prior syphilis infection, which may have determined pathological arterial changes. Moreover, the advanced age of our patient may be considered atypical for a classical, "idiopathic" cervical artery dissection. In the literature, we found one report of a patient with recurrent ischemic strokes and serologic evidence of a previously treated syphilis infection (positive fluorescent treponemal antibody with negative VDRL on both peripheral blood and cerebrospinal fluid), in whom an angiography demonstrated both intracranial and extracranial arterial diseases due to neurosyphilis. ${ }^{10}$ In another case, a fatal vertebrobasilar occlusion occurred in a patient with laboratory findings of a prior spirochetal infection and evidence, at autopsy, of anatomopathological elements suggestive for meningovascular syphilis. ${ }^{11}$

To our knowledge, there are no previous reports in the literature of bilateral ICAD in patients with prior syphilitic infection.

Neurosyphilis still remains an important and treatable cause of stroke in some patients; hence, it would be considered as a part of the diagnostic workup of strokes in patients coming from geographical areas with the highest incidence rates or in individuals with a history of possible exposure to this infection. ${ }^{12}$

\section{Disclosure}

The authors report no conflicts of interest in this work.

\section{References}

1. Wu HC, Chen YC, Chen CJ, Chen ST, Lee TH. Spontaneous bilateral internal carotid artery dissection with acute stroke in young patients. Eur Neurol. 2006;56(4):230-234.

2. World Health Organization. Baseline Report on Global Sexually Transmitted Infection Surveillance. Geneva: WHO; 2012.

3. Ramos TK, Goldstone J. Carotid artery dissection. In: Current Therapy in Vascular Surgery. 3rd ed. Ernst CB, Stanley JC, editor. St Louis: CV Mosby, 1995.

4. Luo H, Liu B, Hu J, Wang X, Zhan S, Kong W. Hyperhomocysteinemia and methylenetetrahydrofolate reductase polymorphism in cervical artery dissection: a meta-analysis. Cerebrovasc Dis. 2014;37(5): 313-322.

5. Rubinstein SM, Peerdeman SM, van Tulder MW, Riphagen I, Haldeman S. A systematic review of the risk factors for cervical artery dissection. Stroke. 2005;36(7):1575-1580.

6. Conde-Sendin MA, AmelaPeris R, Aladro-Benito Y, Maroto AA. Current clinical spectrum of neurosyphilis in immunocompetent patients. Eur Neurol. 2004;52(1):29-35.

7. Clark EG, Danbolt N. The Oslo study of the natural history of untreated syphilis; an epidemiologic investigation based on a restudy of the BoeckBruusgaard material; a review and appraisal. J Chronic Dis. 1955; 2(3):311-344

8. Holmes MD, Brant-Zawadzki MM, Simon RP. Clinical features of meningovascular syphilis. Neurology. 1984;34(4):553-556. 
9. Bourazza A, Kerouache A, Reda R, Mounach J, Mosseddaq R. Meningovascular syphilis: study of five cases. Rev Neurol (Paris). 2008; 164(4):369-373.

10. Aldrich MS, Burke JM, Gulati SM. Angiographic findings in a young man with recurrent stroke and positive fluorescent treponemal antibody (FTA). Stroke. 1983;14(6):1001-1004.

11. Feng W, Caplan M, Matheus MG, Papamitsakis MI. Meningovascular syphilis with fatal vertebrobasilar occlusion. Am J Med Sci. 2009; 338(2):169-171.
12. Cordato DJ, Djekic S, Taneja SR, et al. Prevalence of positive syphilis serology and meningovascular neurosyphilis in patients admitted with stroke and TIA from a culturally diverse population (2005-2009). J Clin Neurosci. 2013;20(7):943-947.

\section{Publish your work in this journal}

Neuropsychiatric Disease and Treatment is an international, peerreviewed journal of clinical therapeutics and pharmacology focusing on concise rapid reporting of clinical or pre-clinical studies on a range of neuropsychiatric and neurological disorders. This journal is indexed on PubMed Central, the 'PsycINFO' database and CAS, and is the official journal of The International Neuropsychiatric Association (INA). The manuscript management system is completely online and includes a very quick and fair peer-review system, which is all easy to use. Visit http://www.dovepress.com/testimonials.php to read real quotes from published authors.

Submit your manuscript here: http://www.dovepress.com/neuropsychiatric-disease-and-treatment-journal 